\title{
A Comparison of Visual Representations of E\&M Plane Waves
}

\author{
Michael Wilson and Robert Beichner \\ Department of Physics, North Carolina State University, 421 Riddick Hall, Raleigh, NC, 27695-8202
}

\begin{abstract}
It is well known that plane waves in electricity and magnetism (E\&M) are misunderstood by students. Particularly, the traditional visual representation of these plane waves is misleading, and students are confused by the waves' three-dimensionality. A possible improvement has been designed using an animated vector field. Graduate physics students were presented two different visual representations of E\&M plane waves. The students were asked to describe what each visual represents in detail. Students' reactions to those two representations are compared and the content delivered in each visualization is assessed, suggesting that the advantages of the animated vector field may be significant.
\end{abstract}

\section{INTRODUCTION}

Electromagnetic waves present a variety of difficulties for students. From polarization and how electric and magnetic fields interact with each other [1] to interference and diffraction [2], student misconceptions about E\&M waves are common [3]. The works of Ambrose and Podolefsky show further that the three-dimensional nature of such waves is especially misunderstood $[4,5]$. This may be partially due the various reasoning and mental models that students employ [6,7] and their difficulties in making connections between those models [8].

In an effort to improve student understanding of electromagnetic waves, modern technology has been applied, introducing interactive simulations of E\&M wave phenomena [9]. This includes innovative interfaces to learn about the propagation of electromagnetic waves [10] and provide researchers with an additional means of studying student difficulties [11]. In addition to technological advances in simulations, success in improving student understanding has been achieved with analogical scaffolding [5], multiple representations [12], and defining a vector field using the divergence and curl [13]. This paper will focus on visualizations of electromagnetic plane waves.

Students in physics often have difficulties in connecting graphs to the physics they represent [14]. This extends to images and graphs of E\&M wave phenomena [15], including the traditional graphical representation of electromagnetic plane waves. In order to better facilitate the understanding of the wave's three-dimensional nature, several novel visual representations were created with a focus on those higher dimensions. Animated vector fields were designed at North Carolina State University [16] and the University of Washington [17], which highlight the wave dynamics and the space that the wave occupies respectively. At Harvard University, a visual was created using parallel planes of constant field value [18]. That visual highlights the uniformity of each cross-section of a plane wave. These potential improvements demonstrate the variety of possible solutions to this problem. As of now, no research has been published addressing the efficacy of any of these visual representations.
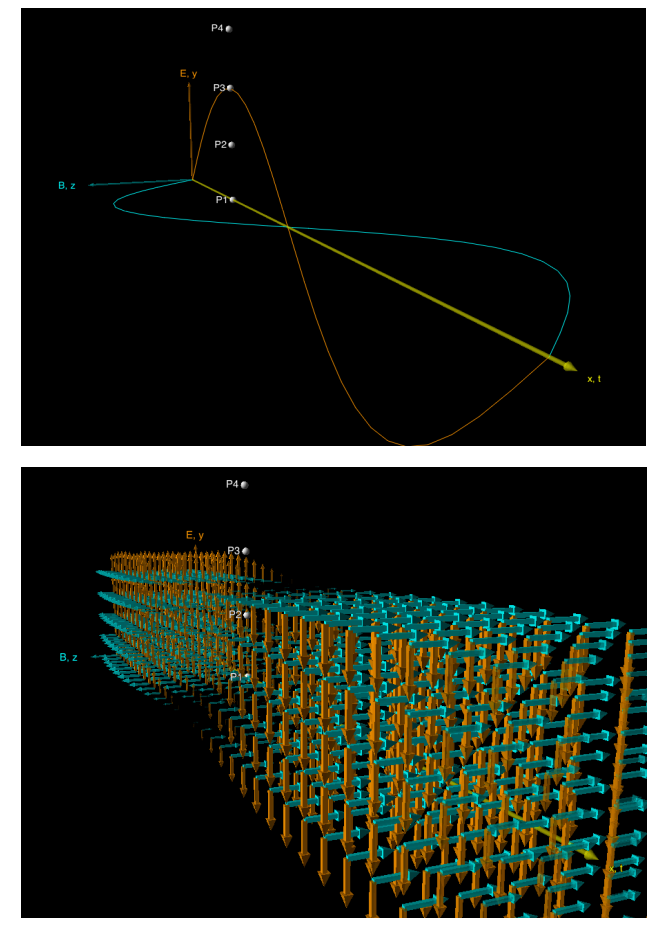

FIG. 1. Traditional Representation (top) and Animated Vector Field (bottom) programmed in VPython [16].

This study uses verbal data from interviews discussing two visualizations of electromagnetic plane waves that were created by Robert Beichner at North Carolina State University [16], shown in Fig. 1. Fifteen volunteer graduate students were shown each of the visuals. The students were split randomly into two groups. Students in one group were shown the traditional representation, asked three questions, then shown the animated vector field and asked the same three questions. This was repeated with the other group, switching the order in which the visuals were shown.

\section{RESEARCH QUESTIONS}

The purpose of this study is to contrast the two visualizations of electromagnetic plane waves shown in Fig. 
1: a traditional representation depicting two static orthogonal sine waves and an animated vector field. Since each student is shown both visualizations, answers can be compared for each of the three questions asked and common responses can be contrasted across visuals.

The first aim of this study is to assess what knowledge is recalled when viewing each visualization. The animated vector field was designed to present more information, so it is interesting to understand how the knowledge claims differ between the two visuals. This gives insight into how effectively such information is portrayed.

Additionally, this study aims to address the effectiveness of each visualization at conveying the idea that a plane wave is a three-dimensional wave with a uniform cross-section. This can be investigated using responses from both the second and third questions for each visual, first with the ranking of the electric field at varying points within the wave and next with the reasoning as to why a given visualization is called a plane wave. Of interest is how complete the answers are, whether they are full or partial descriptions, and what types of misconceptions are prevalent among expert responses. Also, it is interesting how these responses correlate with each other.

It should be noted that any responses given in this study may reflect either what was previously known to the participant or what can be interpreted from the visualization. Since it's difficult to tell which, it is worth comparing answers across the order that visuals are presented and investigate how they change. This gives insight into what may be obtained from the visualizations alone.

\section{METHODOLOGY}

For this study, students who have a working knowledge of electromagnetic plane waves were necessary. This limited the relevant population to students who have completed a second semester physics course, with graduate students better fitting the assumption that participants have expertise on the subject of plane waves. Graduate students are selfselected as top students from their previous schooling, and therefore should have a stronger knowledge base than the average undergraduate physics major.

Interviews were conducted individually, and the audio data was recorded. The students were encouraged to think aloud and give as thorough answers as possible. Half of the participants were shown the traditional representation first, and the other half were shown the animated vector field first. All visualizations were presented on a computer display in full screen mode, and all interview questions were presented in the same manner for consistency's sake. The verbal data was then transcribed by the researcher and segmented into individual clauses.

The first question was to describe what the visualization represents physically. As graduate students, each participant was assumed to have knowledge and understanding about the physics behind the visualizations. This question prompts the students to share some of their expert knowledge and probes which knowledge claims are mediated by the visuals.

The second question was repeated from the work of Ambrose [5]. The participant is asked to rank the strength of the electric field at four specific points within the visualization, the four white points in Fig. 1. It assesses the student's perception of how uniform the field is over a crosssection. The correct answer is that the field is equal at all points for any given instant, but the previous work has shown that incorrect answers are common among all levels of students.

The third question was to describe why the visualization may be referred to as a plane wave. This question probes which knowledge claims are mediated by the visuals, and whether those knowledge claims relate to a plane wave. The most complete response, as with the second question, is that the wave has a uniform cross-section. At any given instant, fields are constant in any plane parallel to the direction of propagation. This description is necessary and sufficient to imply that plane wave properties only vary across one dimension.

Clauses for the interviews were coded according to knowledge topics found within the participants' responses. This was achieved by first tallying up the various student descriptions of each visualization according to the keywords and phrases used, and then grouping these topics into similar bins for study. This led to three coding dimensions comprising of the physics aspects the participants focused on: a description of the type of wave visualized, a description of the motion of that wave, and additional physics topics not directly associated with waves.

Within each of these three dimensions on the participants' knowledge, codes are designed to be hierarchical to be mutually exclusive. The top code in the Wave Type dimension is the "uniform" code which categorizes any clause where the student reasons that the wave has a uniform cross-section. If another Wave Type code could also apply to the same clause, the "uniform" code supersedes it. This applies to every code in descending order in each dimension. Table I shows all codes hierarchal within the Wave Type dimension.

TABLE I. Coding Scheme for Wave Type Dimension.

\begin{tabular}{lll}
\hline \hline \multicolumn{1}{c}{ Codes } & \multicolumn{1}{c}{ Definitions } & \multicolumn{1}{c}{ Examples } \\
\hline Uniform* & $\begin{array}{l}\text { The wave has a uniform } \\
\text { wave front. That is, the } \\
\text { fields and wave } \\
\text { properties don't change } \\
\text { over YZ cross sections }\end{array}$ & $\begin{array}{l}\text { "it's propagating out as a } \\
\text { series of parallel planes" } \\
\text { "Stu05 }\end{array}$ \\
"a cube moving towards \\
you with uniform field \\
strengths" -Stu04 \\
"that's a plane wave" \\
Transverse & The wave is a plane wave & $\begin{array}{l}\text {-Stu01 } \\
\text { "transverse } \\
\text { electromagnetic"-Stu01 }\end{array}$ \\
Other & $\begin{array}{l}\text { The wave is some other } \\
\text { "it looks like it's } \\
\text { type of wave or the type } \\
\text { of wave is unclear }\end{array}$ & -Stu11 \\
\hline \hline $\begin{array}{l}\text { *Most important code in the study. This demonstrates a full understanding } \\
\text { of the wave's three-dimensional nature. }\end{array}$
\end{tabular}




\section{RESULTS}

\section{A. Knowledge Claims by Visual Representation}

The content of the knowledge claims varied little from visual to visual, with only a few exceptions. Differences between the visualizations occurred most strikingly for participants noting a uniform wavefront, noting a onedimensional propagation, and describing the oscillation. Since "uniform" and "1D prop" are the two most important codes when describing why the visual can be referred to as a plane wave, having a difference across visualizations is worth exploring further.

For clauses mentioning the type of wave, participants noted a uniform for the animated vector field $(39 \%, \mathrm{~N}=39)$ at more than double the rate compared to the traditional representation $(15 \%, \mathrm{~N}=12)$. This suggests that the animated vector field does a better job at getting students to deduce that the wave has a uniform cross section than the traditional representation. On the other hand, students noted that the wave traveled in exactly one direction more often when discussing the wave motion of the traditional representation $(25 \%, \mathrm{~N}=21)$ than when discussing the wave motion of the animated vector field $(13 \%, \mathrm{~N}=15)$. This suggests that although the animated vector field might do a better job at getting students to deduce a uniform cross section, the traditional representation may to do a better job at getting students to deduce a one-dimensional propagation.

Knowledge claims noting a uniform wavefront often preceded that of one-dimensional propagation $(45 \%, \mathrm{~N}=10)$ such as with the clause "because it has one plane of oscillation" which is followed by the clause "that plane of oscillation moves with time along the $X$ axis" -Stu01. This occurred more often than the case where a uniform wavefront followed one-dimensional propagation $(22 \%$, $\mathrm{N}=4$ ) such as with the clause "and because this is a plane wave traveling in the X direction" which is followed by the clause "the field strength at each of those four points should be the same" -Stu02. This suggests that understanding the uniform nature of the wave front leads logically to one dimensional propagation more readily than the inverse.

\section{B. Aspects of Understanding by Visual Representation}

In the second question of the dialogue, students were asked to rank the strength of the electric field at several points within the visualization. The correct answer is that the field is equal at all the indicated points. Looking at each student's responses individually and assessing whether or not a code has ever been applied during the dialogue, answers to the second question can be directly compared with whether a student noted a uniform cross-section or onedimensional propagation.

From this, we can see which aspects of a plane wave's three-dimensional nature were more associated with which visualization. The key aspects include a uniform crosssection, one dimensional propagation, and the electric field ranked equal at the four points given. Students who noted both a uniform wavefront and ranked the field equal at all points during the interview were said to have a complete understanding for the purposes of this study.

As shown in Fig. 2, the percentage of participants who noted propagation in one direction and that all points have equal field during the interview didn't vary much across visuals. On the other hand, the percentage of participants that noted a uniform wavefront during the interview was much greater for the animated vector field $(93 \%, \mathrm{~N}=14)$ than the traditional representation $(47 \%, \mathrm{~N}=7)$. This suggests that the uniform wavefront aspect is what varies the most between visualizations. The other aspects do not vary as significantly.

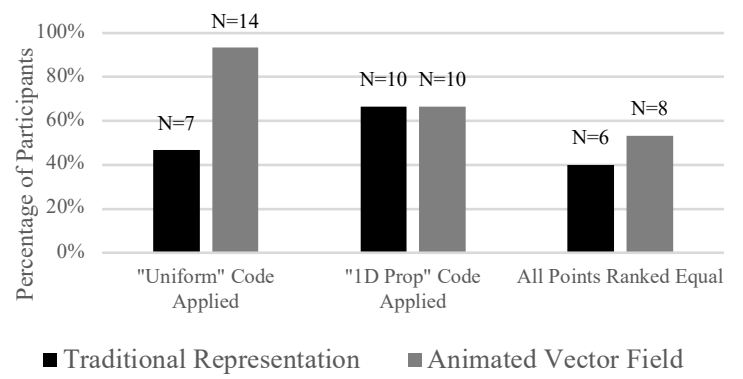

FIG. 2. Aspects of Understanding by Participant

As shown in Fig. 3, the percentage of participants who demonstrated complete understanding also varied between visualizations. $53 \%, \mathrm{~N}=8$ of participants noted both the uniform wavefront and that all points have equal field when discussing the animated vector field. This is true for only $20 \%, \quad \mathrm{~N}=3$ of participants discussing the traditional representation. This suggests that the complete picture is more present in the animated vector field. Furthermore, $33 \%$, $\mathrm{N}=5$ of the participants noted neither of these aspects when discussing the traditional representation. This is true in only one case when discussing the animated vector field. This suggests that in addition to a complete picture, the animated vector field is more likely to at least give a partial understanding.

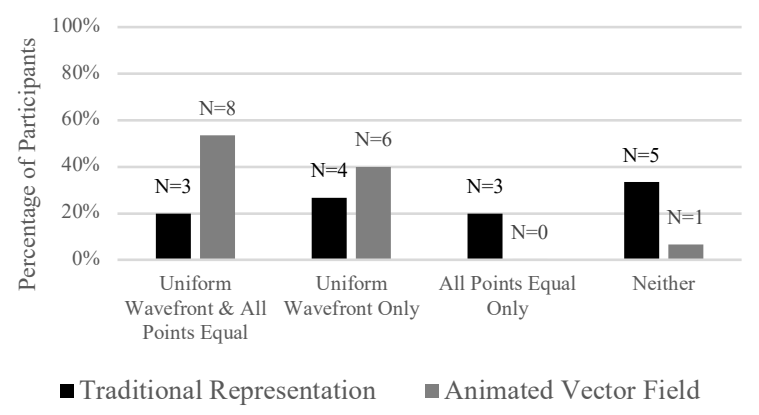

FIG. 3. Co-occurrence of Aspects of Understanding

Further, the percentage of participants who noted a uniform wavefront was much greater for the animated vector field, regardless of the order shown. As shown in Fig. 4, five 
participants who saw the traditional representation first gained the aspect of a uniform wavefront when shown the animated vector field.

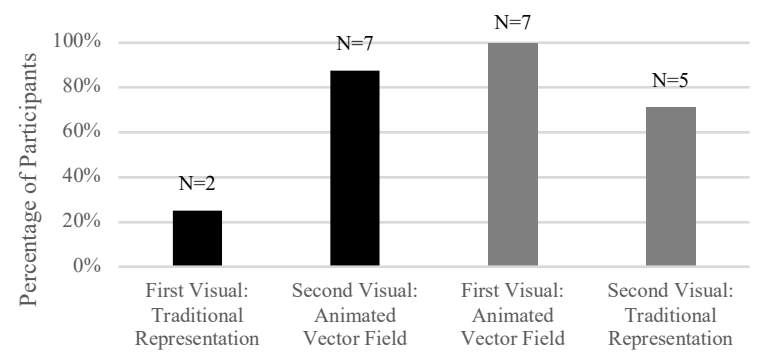

FIG. 4. "Uniform" Code by Order of Visual Shown

Interestingly, when the animated vector field was shown first, two of seven participants failed to carry over the uniform wavefront aspect to the traditional representation. So not only does the animated vector field do a better job at getting students to deduce a uniform wavefront, but this suggests that the traditional representation may even be detrimental to such a deduction. Further investigation is needed for confirmation of this.

\section{DISCUSSION}

This study indicates that the animated vector field representation of an electromagnetic plane wave has advantages over the traditional visualization. Participants more readily picked up on all studied aspects of understanding the wave's three-dimensional nature when discussing the animated vector field compared to discussions of the traditional representation. Given the importance of the concepts involved and the prevalence of difficulty interpreting common instructional representations, further work with larger numbers of students is warranted.

The animated vector field was more likely to prompt a complete understanding of noting a uniform wavefront and ranking the electric field equal at all points within a cross section of the wave. It may also be more likely to prompt at least a partial understanding of noting a uniform wavefront only. Further, the animated vector field prompted this information earlier in the discussion than the traditional representation. Participants showed a more complete understanding and showed it more quickly when discussing the animated vector field. And on two occasions, the traditional representation may have been detrimental to understanding the wave's three-dimensional nature.

A more complete understanding makes sense, as the animated vector field is designed to occupy multiple dimensions and display a uniform wavefront. The visual designed to be better at suggesting a uniform wavefront may be indeed better. But as acknowledged previously, the visual used in this study is not necessarily the only possible means to achieve these results. Visualizations created at the University of Washington [17] and Harvard University [18] highlight the space that the wave occupies and use parallel planes showing constant field value, respectively.

We are working to design three new visualizations that can be used to compare all these various aspects highlighted in each existing visual. This would give insight into what makes a plane wave visualization better than another and give a measure of how well each existing visual does what it is designed to do. From the results of such a study, a possible new and improved solution to this problem may be created which utilizes the most effective aspects of the existing visuals and discards anything students find confusing.

\section{ACKNOWLEDGMENTS}

This project was funded through the North Carolina State University STEM Education Initiative. Special thanks to members of the Physics Education Research and Development Group and Jason Swarts for their help and advice along the way.
[1] M. H. P. Kesonen, M. A. Asikainen and P. E. Hirvonen, Eur. J. Phys. 32, 521 (2011).

[2] K. Wosilait, P. R. L. Heron, P. S. Shaffer, and L. C. McDermott, Am. J. Phys. 67, S5 (1999).

[3] D. P. Maloney, T. L. O'Kuma, C. J. Hieggelke, and A. van Heuvelen, Am. J. Phys. 69, S12 (2001).

[4] B. S. Ambrose, P. R. L. Heron, S. Vokos, and L. C. McDermott, Am. J. Phys. 67, 891 (1999).

[5] N. S. Podolefsky and N. D. Finkelstein, Phys. Rev. ST Phys. Educ. Res. 3, 010109 (2007).

[6] L. Maurines, Int. J. Sci. Educ. 32(14), 1895 (2010).

[7] S. K. Şengören, J. Balt. Sci. Educ. 9(1), 61 (2010).

[8] P. Colin and L. Viennot, Am. J. Phys. 69, S36 (2001).

[9] M. F. Iskander, IEEE T. Microw. Theory, 50(3), 1015 (2002).

[10] F. Sagnard, IEEE T. Educ. 47(3), 389 (2004).
[11]D. Zarras and C. Solomonidou, Proc. IASTED Int. Conf. Comput. Adv. Technol. Educ. 10, 158 (2007).

[12] J. A. Bryan and B. D. Fennell, Phys. Educ. 44, 403 (2009).

[13] B. C. Xue, R. L. C. Hazelton, P. S. Shaffer, and P. R. L. Heron, presented at the Physics Education Research Conference 2016, Sacramento, CA, 2016.

[14] L. C. McDermott, M. L. Rosenquist, and E. H. van Zee, Am. J. Phys. 55, 503 (1987).

[15] P. Colin, F. Chauvet, and L. Viennot, Int. J. Sci. Educ. 24(3), 313 (2002).

[16] R. Beichner, computer code VPython, North Carolina State University, Raleigh, NC, 2012.

[17] X. Cid and R. Lopez, computer code GlowScript, University of Washington, Seattle, WA, 2016.

[18] E. Mazur, Principles and Practice of Physics 1st Edition, (Pearson Education 2015), p. 805. 